Document downloaded from:

http://hdl.handle.net/10251/51218

This paper must be cited as:

Ruiz Calvo, F.; Rosa, MD.; Acuña, J.; Corberán Salvador, JM.; Montagud Montalvá, Cl. (2015). Experimental validation of a short-term Borehole-to-Ground (B2G) dynamic model. Applied Energy. 140:210-223. doi:10.1016/j.apenergy.2014.12.002.

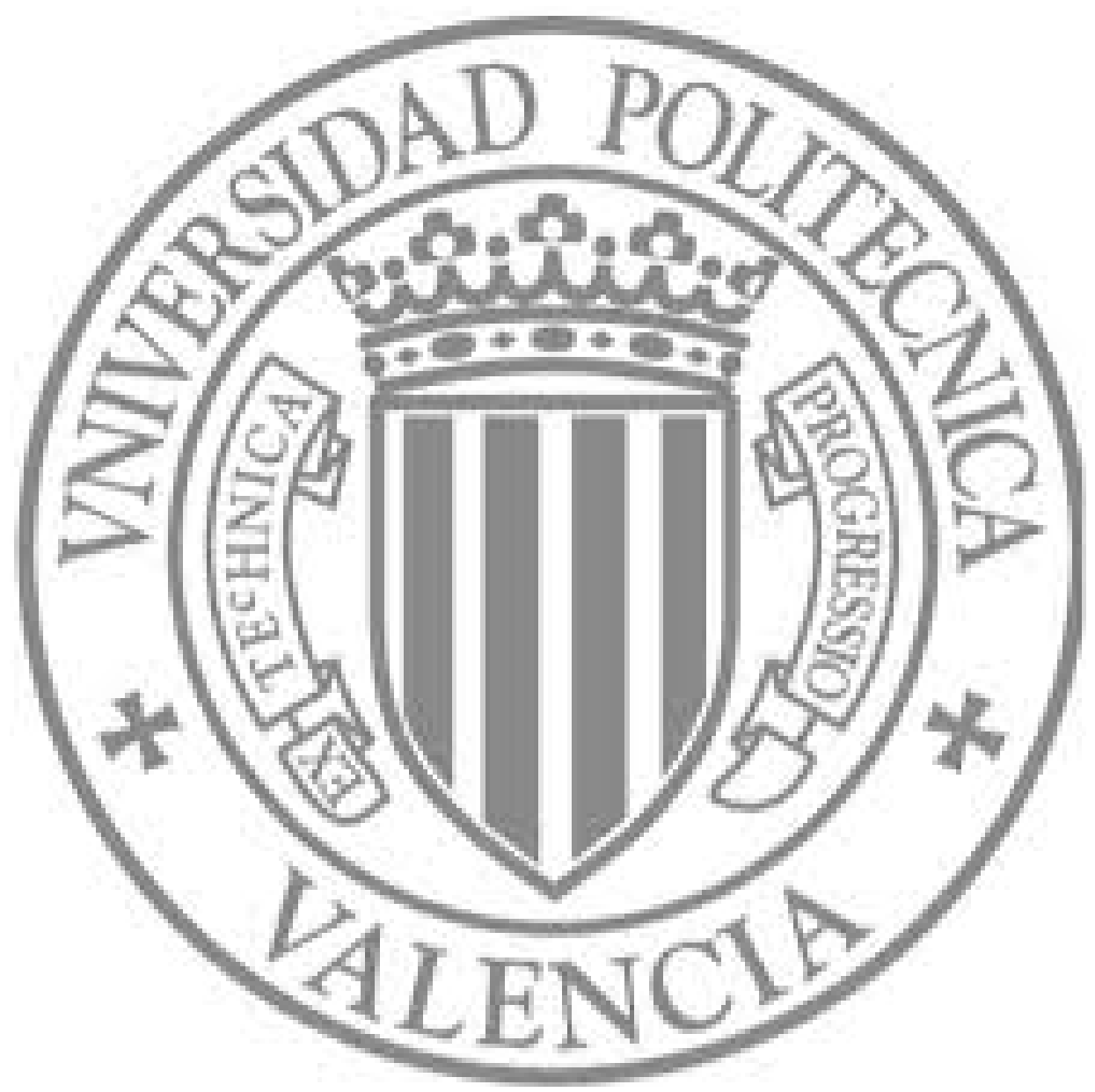

The final publication is available at

http://dx.doi.org/10.1016/j.apenergy.2014.12.002

Copyright Elsevier 


\title{
Experimental validation of a short-term Borehole-to-Ground (B2G) dynamic model
}

\author{
F. Ruiz-Calvo ${ }^{a, *}$, M. De Rosab ${ }^{\mathrm{b}}$, J. Acuña ${ }^{\mathrm{c}}$, J.M. Corberán $^{\mathrm{a}}$, C. Montagud ${ }^{\mathrm{a}}$ \\ ${ }^{a}$ Instituto de Ingeniería Energética, Universitat Politècnica de València. \\ Camino de Vera sn 46022 Valencia, Spain. Phone: 34-96-3879910. Fax: 34-963877272 \\ ${ }^{b}$ DIME/TEC - Division of Thermal Energy and Environmental Conditioning, \\ University of Genoa. Via All'Opera Pia, 15/A, 16145 Genoa, Italy. \\ ${ }^{c}$ KTH, Royal Institute of Technology, Brinellvägen 68, Stockholm
}

\begin{abstract}
The design and optimization of ground source heat pump systems require the ability to accurately reproduce the dynamic thermal behavior of the system on a short-term basis, specially in a system control perspective. In this context, modelling borehole heat exchangers (BHEs) is one of the most relevant and difficult tasks. Developing a model that is able to accurately reproduce the instantaneous response of a BHE while keeping a good agreement on a long-term basis is not straightforward. Thus, decoupling the short-term and long-term behavior will ease the design of a fast shortterm focused model. This work presents a short-term BHE dynamic model, called Borehole-to-Ground (B2G), which is based on the thermal network approach, combined with a vertical discretization of the borehole.

The proposed model has been validated against experimental data from a real borehole located in Stockholm, Sweden. Validation results prove the ability of the model to reproduce the short-term behavior of the borehole with an accurate prediction of the outlet fluid temperature, as well as the internal temperature profile along the U-tube.
\end{abstract}


Keywords: ground source heat pump, borehole heat exchanger, heating and cooling systems, dynamic modelling

\section{Introduction}

2 Ground source heat pumps (GSHPs) represent one of the common avail3 able and profitable geothermal systems to provide space conditioning in a 4 wide range of applications [1. A typical GSHP consists of a heat pump 5 coupled with a ground heat exchanger (GHE), which permits to utilize the 6 ground as a heat source in winter and as a heat sink in summer. Different 7 configurations can be adopted, but one of the most commonly used is the

8 borehole heat exchanger (BHE) in which one or several boreholes are drilled

9 vertically in the soil, allowing the heat exchange between the heat carrier 10 fluid and the ground. A detailed review of GHE systems can be found in 11 [.

In order to optimize a GSHP system as well as to improve the design, 13 special attention should be paid to the analysis of the interaction between 14 the heat pump and the ground source heat exchanger. In the last years 15 many researchers focused their attention on the GSHP systems with BHE, 16 performing both experimental and theoretical studies in order to evaluate 18 [3-14])

${ }^{*}$ Corresponding author at: Instituto de Ingeniería Energética, Universitat Politècnica de València. Camino de Vera sn 46022 Valencia, Spain. Phone: 34-96-3879120. Fax: 34963877272

Email addresses: fliruica@etsii.upv.es (F. Ruiz-Calvo), mattia.derosa@unige.it (M. De Rosa), jose.acuna@energy.kth.se (J. Acuña), corberan@upvnet.upv.es (J.M. Corberán), carmonmo@iie.upv.es (C. Montagud) Preprint submitted to Applied Energy 
In this context, software able to predict the BHE thermal performance

The most important limitation of the g-function approaches is that they are valid only for a time scale greater than $t_{b}$ (Eq. 1), resulting in 3 to 6 hours for a typical borehole [25].

$$
t_{b}=5 \frac{r_{b}^{2}}{\alpha}
$$


40 Another approach to numerically describe a vertical borehole is the ther-

41 mal network model, in which the borehole and the surrounding ground are

42 represented as a series of temperature nodes connected by thermal resis-

43 tances. The basic thermal network is the steady-state delta network [25] in

44 which one temperature node is located on each pipe of the $U$ tube and one

45 temperature node is located on the borehole wall (Figure 1).

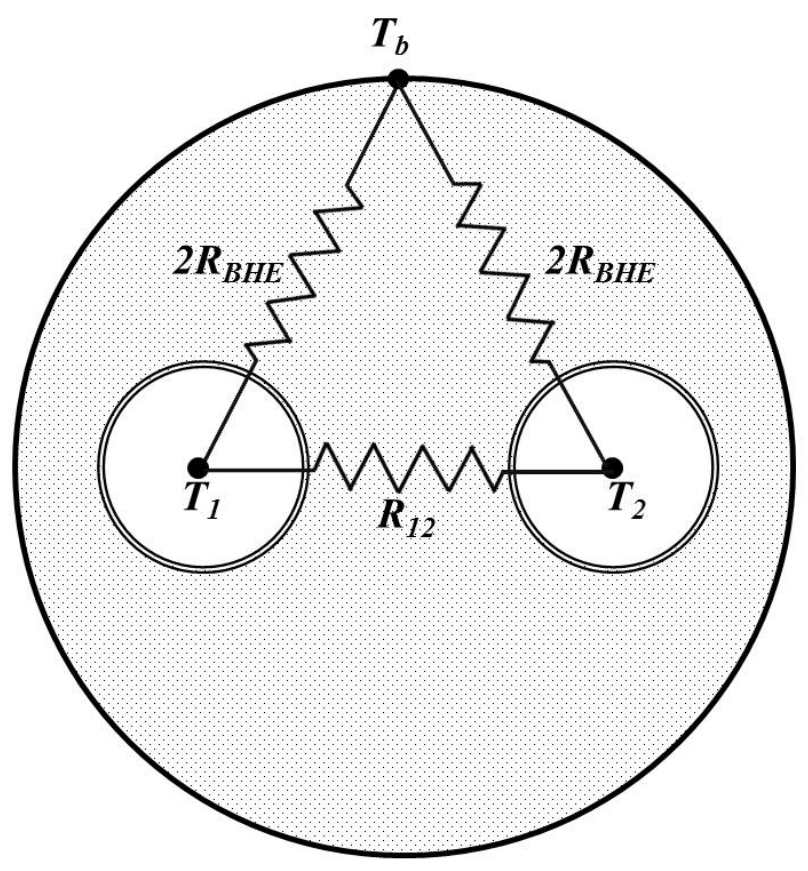

Figure 1: Standard steady state delta network 25.

46 Generally, short-term regulation criteria assume an important role in 47 the global energy performance of systems, especially when different energy 48 sources are coupled together. In the global context of the global mod49 elling of GSHP installations, it is necessary to consider that many of them so are based on an $\mathrm{ON} / \mathrm{OFF}$ regulation criterion, in which the thermal load 
51 is injected/extracted to the ground in short heat pulses with a duration 52 of about 20 minutes (depending on the instantaneous thermal energy de53 mand), introducing strong dynamic components on the evolution of the ${ }_{54}$ fluid temperatures. Therefore, the short-term behavior of BHEs represents 55 a crucial topic for evaluating and optimizing the thermal performance of 56 GSHPs, especially considering that the fluid temperature evolution is a key 57 parameter due to its strong influence on the heat pump performance and 58 since the possible control algorithms are mostly based on it.

59 In this context, models able to accurately predict the evolution of the 60 BHE fluid temperatures on a short-term basis can be very useful for op61 timization purposes. Several attempts have been performed in order to overcome the steady-state approach introducing the BHE dynamic perfor63 mance. Yang et al. 26] proposed a two-region analytical solution model for 64 vertical U-tube: the cylindrical heat source theory is adopted for the out65 side soil region in order to calculate the borehole wall temperature, while 66 the outlet fluid temperature is calculated by modelling the heat transfer 67 inside the borehole in steady state conditions by using the standard delta 68 network [25]. The model has been validated against experimental data of 69 about 120 hours of operation, showing a good agreement especially in a long-term perspective. Despite that, as stated by the authors, the steady 71 state assumption in modelling the heat transfer inside the borehole affects 72 the model accuracy during the first 7 hours of simulation.

73 In order to model the short-term response Javed and Claesson 27] devel74 oped an analytical solution based on the assumption that the heat transfer 75 inside the borehole is completely radial and modeling the U-tube using an 76 equivalent diameter with a single average temperature. The analytical so- 
77 lution was derived by solving the problem in the Laplace domain and it was 78 validated by comparing its results with ones obtained by other numerical 79 and analytical models and against experimental data. A similar approach is 80 proposed by Monteyne et al. [28] with the aim to develop a new procedure 81 for performing BHE thermal response test (TRT). The borehole heat trans82 fer is modeled adopting a frequency domain model: the relation between 83 the heat and the temperature is approximated using a rational Frequency ${ }_{84}$ Response Function (FRF) in the Laplace variable $(s)$ or in the Warburg $85 \operatorname{variable}(\sqrt{s})$ in order to calculate the outlet water temperature at each ${ }_{86}$ sequence of heat injection pulse. The TRT procedure has been validated 87 against experimental data showing a good performance. Li et al. [29] modi88 fied the original line-source theory developing a modified response functions 89 (G-function) in order to model the short-term heat conduction problem both 90 for pile with spiral coils and for BHE with U-tubes. Authors stated that 91 the new $\mathrm{G}$ function permits to model BHE in a time scale between one hour 92 to several years and it can be useful for annual energy analyses of GSHP ${ }_{93}$ system. An extension of this work is provided in [30] in which the model 94 has been validated against experimental data for short-term periods. Au-

Starting from the work performed by [25, Bauer et al. 31] modified the standard thermal network introducing several temperature nodes for the internal grout zone subdivided in two or more different layers, depending on the tube geometry, and lumping the correspondent thermal capacitance in each layer. Moreover, a one-dimensional radial finite difference description 
of the surrounding ground was adopted. Since then, many improvements have been made to the delta network, usually adding more nodes to the network, as in [32] and [33], or dividing the borehole in two or more areas, depending on the internal borehole geometries [34]. In this context, the borehole thermal resistance assumes a relevant issue, since it represents the resistance between the pipes and the borehole wall. It can be experimentally obtained or it can be calculated analytically. An exhaustive review of different methods to obtain the borehole resistance is provided in Lamarche et al. 34].

Finally, the finite elements model (FEM) represents one of the more detailed approaches, directly solving the three-dimensional heat transfer problem, despite a high computational costs due to the more detailed discretization of the borehole and of the surrounding ground. Therefore, FEMs are usually assumed as a reference for numerical analysis or validation of simplified models that can provide faster results, although not being so accurate (as in [31, 32], etc.). Some examples of FEM can be found in [35 42]. In particular, Koohi-Fayegh et al. [39] used the FEM approach to investigate the effect of the system performance due to the thermal interaction of different BHEs. Following the same approach suggested by [35, 36], Florides et al. 40] developed and validated a numerical model which combined a 3D conduction in the soil, solved using a FEM approach, with a 1D modeling of the carrier fluid. The model was implemented in the FlexPDE environment and it was validated against experimental data showing good results. In a subsequent work [41], the model has been extended to single and double U-tube BHE with multiple-layer soil substrate. Recently, Luo et al. [42] utilized a FEM approach in order to investigate the thermal performance 
of three groups of BHEs buried in a soil with different geological layers.

Therefore, most of the currently available models are focused on longterm response, while models able to predict the BHE short-term behaviour are usually based on FEM technique, which introduces high computational costs. Generally, it is difficult to obtain a model which can be used for simulation of both short-term and long-term behavior with a computational cost low enough in order to combine the model with other component ones, especially considering that the currently available models simultaneously calculate the local and global solutions. The novelty of the proposed approach consists in using two separate models for the local and global solution calculation, decoupling the short-term and long-term simulation and allowing the use of faster models on each side. Thus, the short-term model only takes into account the local heat transfer between the fluid flow, the borehole and its adjacent piece of ground. Considering the GSHP typical operation, this short-term model should be able to reproduce the instantaneous performance of the BHE during the daily heat injection/extraction times up to 10 hours in a ON-OFF operating control criteria, starting from the initial ground temperature of each day. Then, the long-term model should be able to calculate the initial ground temperature for each day, taking into account the thermal load of the previous one. This should reduce the total computational cost of the whole model, since it is not necessary to calculate the long-term response of the ground at every time-step.

In this paper the short-term BHE dynamic model, called Borehole-toGround (B2G) model, is presented. The model is based on the thermal network approach, keeping the number of nodes as low as possible while still being able to simulate the short-term (10 to 15 hours) behavior with- 
out an excessive computational cost, accurately predicting not only the final temperature values but also the instantaneous response of a BHE. Besides, B2G model is presented as a user-friendly simulation tool which can be easily calibrated and adapted to different single U-tube BHEs and can be implemented in all computational environments. For this purpose a detailed description of the model is reported in section 2. The model has been implemented in TRNSYS environment and it has been validated against experimental data measurements collected during two step-test for a single U-tube borehole located in Stockholm, Sweden. A comparison of the performance of $\mathrm{B} 2 \mathrm{G}$ with that of an standard steady-state model in a real ON/OFF GSHP operation can be found in [43].

\section{B2G dynamic model}

\subsection{Model description}

A short-term BHE dynamic model, called Borehole-to-Ground (B2G) model, has been developed, based on previous works [26, 27, 31 34]. The model is intended to correctly predict the behavior of U-tube boreholes in terms of water temperature throughout the pipe for short-term periods. Starting from the work carried out by Bauer et al. [31, 32], a vertical discretization of the borehole is performed and, for each node, a thermal network is proposed that describes the radial heat transfer at each borehole depth (Figure 2a). The thermal network configuration has been chosen in order to ensure a good accuracy of the model predictions while reducing the total number of parameters as much as possible. As a result, five thermal capacitances and six thermal resistances are taken into account at each depth (5C6R-n model, where $\mathrm{n}$ is the number of the nodes), considering 
180 the thermal properties of the ground, the grout and the pipes (Figure 2b).

181 Vertical heat conduction is neglected, leading to the following statements:

- for the fluid nodes, taking into account the vertical direction advection and the heat exchange with the correspondent grout node and with the adjacent fluid node, the transient energy balance equations result in equations 2 and 3 .

$$
\begin{aligned}
& \frac{\partial T_{1}(z)}{\partial t}=-v \frac{\partial T_{1}(z)}{\partial z}-\frac{1}{C_{f}}\left(\frac{T_{1}(z)-T_{b 1}(z)}{R_{b 1}}+\frac{T_{1}(z)-T_{2}(z)}{R_{p p}}\right) \\
& \frac{\partial T_{2}(z)}{\partial t}=-v \frac{\partial T_{2}(z)}{\partial z}-\frac{1}{C_{f}}\left(\frac{T_{2}(z)-T_{b 2}(z)}{R_{b 2}}-\frac{T_{1}(z)-T_{2}(z)}{R_{p p}}\right)
\end{aligned}
$$

- for the grout inside the borehole, two separate regions are considered, as shown in Figure 2a, resulting in two different grout nodes 34 with a lumped thermal capacitance. Both nodes are interconnected by a thermal resistance $R_{b b}$, and to a common ground node by the resistance $R_{g}$, resulting in a delta-network different from the standard delta-network [19], which is limited to the internal borehole geometry, as shown in Figure 11). Equations 4 and 5 correspond to the energy balance equations for both grout nodes.

$$
\begin{gathered}
C_{b 1} \frac{\partial T_{b 1}(z)}{\partial t}=\frac{T_{1}(z)-T_{b 1}(z)}{R_{b 1}}+\frac{T_{b 1}(z)-T_{b 2}(z)}{R_{b b}}-\frac{T_{b 1}(z)-T_{g}(z)}{R_{g}} \\
C_{b 2} \frac{\partial T_{b 2}(z)}{\partial t}=\frac{T_{2}(z)-T_{b 2}(z)}{R_{b 1}}-\frac{T_{b 1}(z)-T_{b 2}(z)}{R_{b b}}-\frac{T_{b 2}(z)-T_{g}(z)}{R_{g}}
\end{gathered}
$$


- the last node in the thermal network at each z-depth corresponds to the ground node $T_{g}$, which is connected with the two grout nodes $\left(T_{b 1}\right.$ and $T_{b 2}$ ) by the same thermal resistance $R_{g}$ (Eq. 6).

$$
C_{g} \frac{\partial T_{g}(z)}{\partial t}=\frac{T_{b 1}(z)-T_{g}(z)}{R_{g}}+\frac{T_{b 2}(z)-T_{g}(z)}{R_{g}}
$$

a)

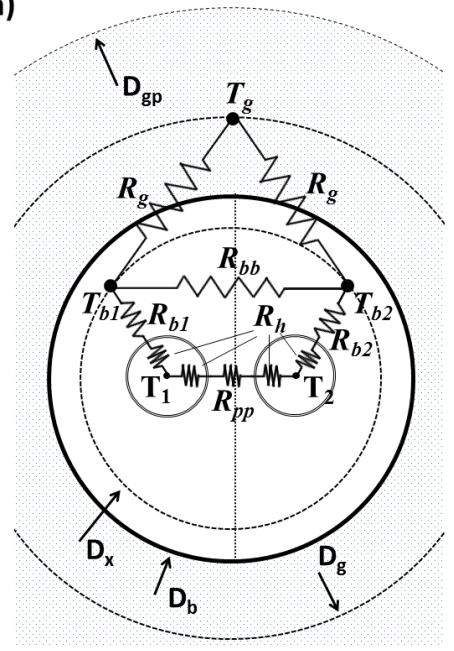

b)

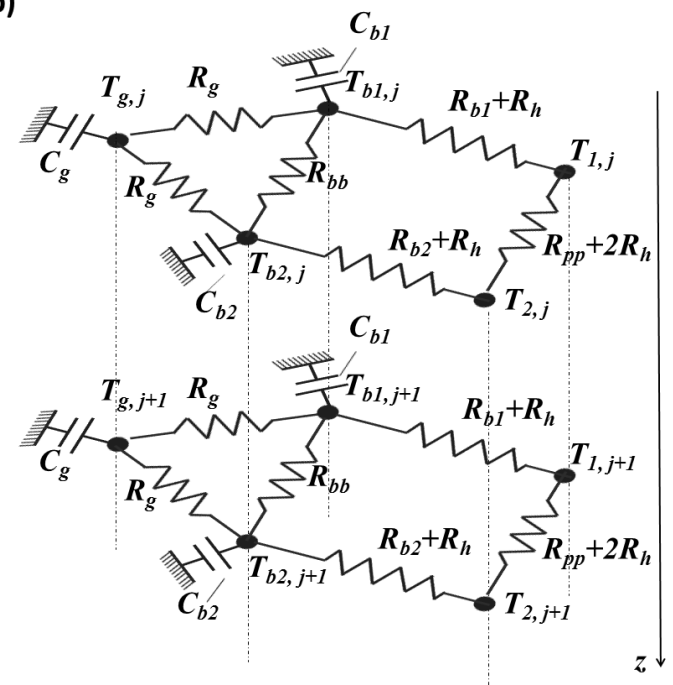

Figure 2: Thermal network model adopted in the present work: a) 2D model; b) 3D model.

Equations 2 to 6 conform a system of ordinary differential equations, which are solved using standard numerical techniques (see section 2.3).

As stated in section 1, the aim of $\mathrm{B} 2 \mathrm{G}$ is to provide an accurate prediction of the short-term behavior with a reduced computational cost. The thermal network suggested in this approach is slightly different from those found in literature, since it divides the grout zone into two separate nodes and situates the delta network between those nodes and the one located in 


\section{Thermal capacitances.}

First, the thermal capacitances $C_{b 1}$ and $C_{b 2}$ are calculated considering the volume of each grout zone, following Eq. 7 and Eq. 8 .

$$
\begin{gathered}
C_{b 1}=C_{b 2}=\mathrm{d} z \cdot\left(\frac{S_{b}}{2} c_{b}+S_{p} c_{p}\right) \\
S_{b}=\frac{\pi}{4}\left(D_{b}^{2}-2 D_{p, e}^{2}\right)
\end{gathered}
$$

In these equations, $S_{b}$ is the borehole section neglecting the pipes, $D_{p, e}$ is the external pipe diameter, $\mathrm{d} z$ is the node length and $c_{b}$ is the grout volumetric heat capacity. The thermal capacitance of the pipe walls is small when compared to that of the grout, so, the term $S_{p} c_{p}$ can be neglected in equation 7, resulting in equation 9

$$
C_{b 1}=C_{b 2} \approx \mathrm{d} z \cdot \frac{S_{b}}{2} c_{b}
$$


Thermal resistances.

The thermal resistances between the grout and pipe nodes depend on the overall borehole thermal resistance $R_{B H E}$. This resistance is the average thermal resistance between the circulating fluid and the borehole wall, as represented in Figure 3 a. Usually, this parameter is determined by experimental tests.

a)

b)

c)

d)
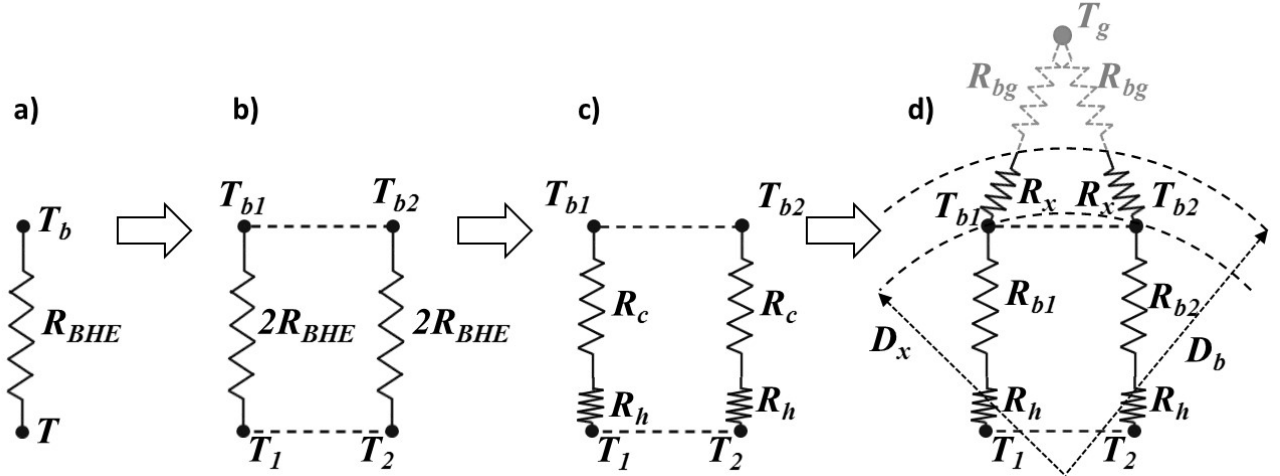

Figure 3: Thermal resistances definition steps: a) borehole resistance, b) parallel borehole resistances, c) convective and conductive resistances, d) final resistances configuration.

Since the grout zone has been divided into two nodes, $R_{B H E}$ has to be divided into two thermal parallel resistances which connect each pipe with the corresponding grout zone, as shown in Figure 3b. Besides, as shown in Figure 3c, each one of these parallel resistances can be separated into a convective $\left(R_{h}\right)$ and a conductive term $\left(R_{c}\right)$ (Eq. 10).

$$
2 R_{B H E}=R_{h}+R_{c}
$$

The conductive thermal resistance on equation $10, R_{c}$, accounts for the total conductive resistance between the pipes and the borehole wall. However the grout nodes will be located somewhere in between them, at a certain 
diameter $D_{x}$. Therefore, $R_{c}$ is divided into two different resistances (Figure 3d), following Eq. 11.

$$
\begin{gathered}
\qquad R_{c}=R_{b}+R_{x} \\
\text { where } \quad R_{b}=R_{b 1}=R_{b 2}
\end{gathered}
$$

${ }_{210}$ The resistance between the grout nodes and the borehole wall $\left(R_{x}\right)$ will ${ }_{211}$ be added to the ground thermal resistance $R_{b g}$ (Figure $3 \mathrm{~d}$ ), in order to ${ }_{212}$ calculate the parameter $R_{g}$ from the thermal network (Figure 2) as shown in ${ }_{213}$ Eq. 12 . The thermal resistance $R_{b}$ corresponds to the conductive resistance ${ }_{214}$ from the pipes to each grout node, which is the one represented by the ${ }_{215}$ parameters $R_{b 1}$ and $R_{b 2}$ from the thermal network (Figure 2).

$$
R_{g}=R_{b g}+R_{x}
$$

The convective term $R_{h}$ from Eq. 10 can be calculated as follows:

$$
R_{h}=\frac{1}{\pi D_{p, i} \mathrm{~d} z h}=\frac{1}{\pi \mathrm{d} z \mathrm{Nu} k}
$$

216 where $D_{p, i}$ is the internal pipe diameter, and $N u$ is the Nusselt number 217 which can be calculated according to [45].

${ }_{218}$ The global borehole thermal resistance $R_{B H E}$ can be obtained by means ${ }_{219}$ of several experimental step-tests, and then the different terms presented in 220 equations 10 and 11 can be obtained from the experimental $R_{B H E}$. Oth221 erwise, it is possible to estimate it theoretically. One of the most common 222 calculation methods is to establish an equivalent representative surface $S_{e q}$ ${ }_{223}$ (Figure 4 a), which provides an equivalent diameter $D_{e q}$, according to equa224 tion 14 .

$$
D_{e q}=2 \sqrt{\frac{S_{e q}}{\pi}}
$$


There are different approaches to the estimation of the equivalent surface. Pasquier et al. [33] suggest to consider the sum of $S_{g g}$ and $S_{p}$ surfaces, as shown in Figure 4 a. Therefore, the equivalent diameter will be calculated following the equation 15 .

$$
D_{e q}=D_{p, e} \sqrt{\frac{4 W}{\pi D_{p, e}}+1}
$$

This allows the calculation of both conductive thermal resistances $\left(R_{x}\right.$ and $R_{b}$ ) considering a semi-cylindrical conductive heat transfer (Figure $4 \mathrm{~b}$ ), following equations 16 and 17 , where $k_{b}$ is the thermal conductivity of the grout.

$$
\begin{gathered}
R_{b}=R_{b 1}=R_{b 2}=\frac{\ln \left(D_{x} / D_{e q}\right)}{\pi k_{b} \mathrm{~d} z} \\
R_{x}=\frac{\ln \left(D_{b} / D_{x}\right)}{\pi k_{b} \mathrm{~d} z}
\end{gathered}
$$

It should be pointed out that the position of the two grout nodes can strongly affect the performance of the model, since the values of the conductive thermal resistances directly depend on it. The position $D_{x}$ (with $\left.D_{e q}<D_{x}<D_{b}\right)$ depends on the internal borehole geometry, especially on the position of the U-tube pipes, the shank spacing and the distance between the pipes and the borehole wall. Therefore, determining the position $D_{x}$ is not straightforward. It seems reasonable to think that, when the pipes are close to the borehole wall, it is preferable to locate the grout nodes on the borehole wall, giving $D_{x}=D_{b}$. In other cases, a sensitivity analysis on the effect of different values of $D_{x}$ can be performed in order to obtain a useful approximation.

Finally, there are two more thermal resistances on the thermal network that depend on the grout properties. Obtaining the thermal resistance be- 


\subsubsection{Ground node}

For the ground node, both the thermal capacitance $C_{g}$ and thermal resistance $R_{b g}$ depend on the penetration depth $D_{g p}$ of the borehole which, in turn, depends on the heat injection/extraction time and on the ground thermal diffusivity [19]. For a given penetration depth, the thermal capacitance $C_{g}$ can be calculated from Eq. 20.

$$
C_{g}=\frac{\pi}{4}\left(D_{g p}^{2}-D_{b}^{2}\right) c_{g} \mathrm{~d} z
$$

For the calculation of the ground thermal resistance $R_{b g}$, a diameter $D_{g}$ can be calculated as the mean diameter between the borehole $D_{b}$ and the penetration diameter $D_{g p}$. The ground capacitance nodes $C_{g}$ are considered to be lumped in this diameter, allowing the calculation of the thermal 

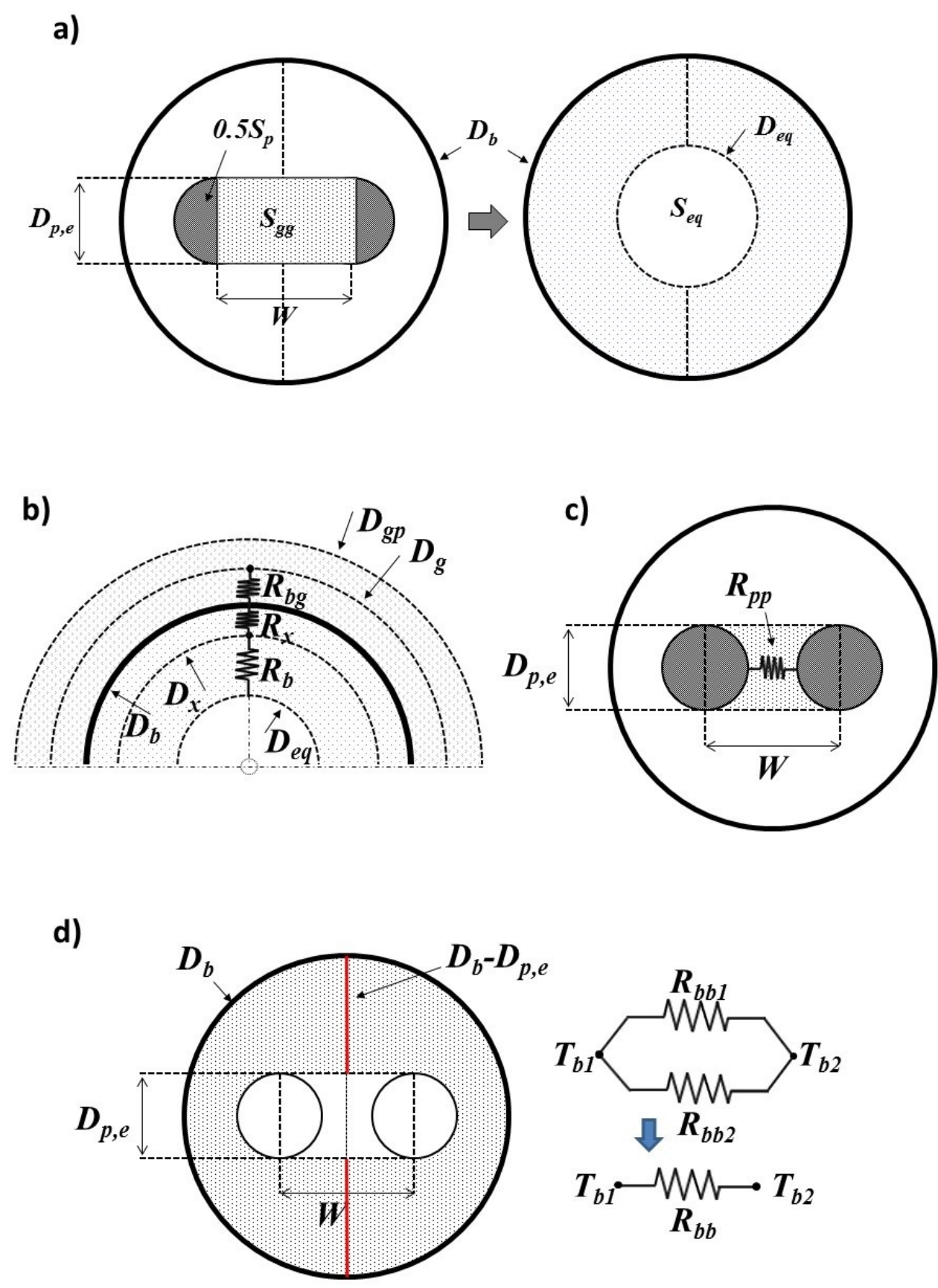

Figure 4: Geometrical model characteristics to calculate a) the equivalent diameter [33, b) grout nodes position, c) pipe to pipe therpnal resistance, d) grout node to grout node thermal resistance. 
resistance as a cylindrical conductive heat transfer, following Eq. 21.

$$
R_{b g}=\frac{1}{\pi k_{g} \mathrm{~d} z} \ln \left(\frac{D_{g}}{D_{b}}\right)
$$

Finally, the total thermal resistance $R_{g}$ between the grout nodes and the ground node as previously considered in Eqs. 446 can be calculated according to Eq. 22 .

$$
R_{g}=R_{x}+R_{b g}
$$

$$
\begin{aligned}
T_{1}^{n+1}(j)= & T_{1}^{n}(j)-\frac{\Delta t v}{2 \mathrm{~d} z}\left(\left(T_{1}^{n}(j+1)-T_{1}^{n}(j-1)\right)-\frac{\Delta t v}{\mathrm{~d} z}\left(T_{1}^{n}(j+1)-2 T_{1}^{n}(j)+T_{1}^{n}(j-1)\right)\right) \\
& -\frac{\Delta t}{C_{f}}\left(\frac{T_{1}^{n}(j)-T_{b 1}^{n}(j)}{R_{b 1}}+\frac{T_{1}^{n}(j)-T_{2}^{n}(j)}{R_{p p}}\right)
\end{aligned}
$$




$$
\begin{gathered}
T_{2}^{n+1}(j)=T_{2}^{n}(j)-\frac{\Delta t v}{2 \mathrm{~d} z}\left(\left(T_{2}^{n}(j+1)-T_{2}^{n}(j-1)\right)-\frac{\Delta t v}{\mathrm{~d} z}\left(T_{2}^{n}(j+1)-2 T_{2}^{n}(j)+T_{2}^{n}(j-1)\right)\right) \\
-\frac{\Delta t}{C_{f}}\left(\frac{T_{2}^{n}(j)-T_{b 2}^{n}(j)}{R_{b 2}}-\frac{T_{1}^{n}(j)-T_{2}^{n}(j)}{R_{p p}}\right) \\
T_{b 1}^{n+1}(j)=T_{b 1}^{n}(j)+\frac{\Delta t}{C_{b 1}}\left(\frac{T_{1}^{n}(j)-T_{b 1}^{n}(j)}{R_{b 1}}+\frac{T_{b 1}^{n}(j)-T_{b 2}^{n}(j)}{R_{b b}}-\frac{T_{b 1}^{n}(j)-T_{g}^{n}(j)}{R_{g}}\right) \\
T_{b 2}^{n+1}(j)=T_{b 2}^{n}(j)+\frac{\Delta t}{C_{b 2}}\left(\frac{T_{2}^{n}(j)-T_{b 2}^{n}(j)}{R_{b 2}}-\frac{T_{b 1}^{n}(j)-T_{b 2}^{n}(j)}{R_{b b}}-\frac{T_{b 2}^{n}(j)-T_{g}^{n}(j)}{R_{g}}\right) \\
T_{g}^{n+1}(j)=T_{g}^{n}(j)+\frac{\Delta t}{C_{g}}\left(\frac{T_{b 1}^{n}(j)-T_{g}^{n}(j)}{R_{g}}+\frac{T_{b 2}^{n}(j)-T_{g}^{n}(j)}{R_{g}}\right)
\end{gathered}
$$

260 The time-step $(\Delta t)$ used for the calculations depends on the time-step of

261

262

263

264

265

266

267

268

269

the simulation, and its maximum value is fixed by the Courant-Friedrichs-

Lewy (CFL) condition (Eq 28).

$$
\begin{aligned}
\frac{\Delta t}{\Delta t_{M A X}}=C F L & \leq 1 \\
\text { where } \Delta t_{M A X} & =\frac{\mathrm{d} z}{v}
\end{aligned}
$$

Finally, if the simulation time-step is greater than $\Delta t_{M A X}$ given by Eq. 28, it will be necessary to subdivide it into smaller time-steps that satisfy the CFL condition.

\section{Validation}

\subsection{KTH Borehole}

The data used for the model validation have been collected during distributed thermal response tests (DTRTs) carried out in a $260 \mathrm{~m}$ deep water 
filled borehole installed in Sweden. The borehole diameter is $140 \mathrm{~mm}$ and the groundwater level was $5.5 \mathrm{~m}$. The U-tube is made of a PE pipe 40x2.4 $\mathrm{mm}$ with a total length of $257 \mathrm{~m}$. The working fluid is an aqueous solution of $12.6 \%$ weight concentration ethanol. Distributed temperature sensing technique is implemented in the borehole in order to record the groundwater and fluid temperatures at different depths, measured every meter.

Two different tests have been considered with different constant mass flow rates $(0.50$ and $0.44 \mathrm{l} / \mathrm{s})$. The duration of the first test considered extended up to 160 hours with 24 hours of pre-circulation without heat injection and about $48 \mathrm{~h}$ of constant heat injection. As reported in [47, the results of this test allow the calculation of the mean borehole thermal resistance and the ground (rock) thermal conductivity, which will be used in the next sections. A second test has been performed where, after about 70 hours of pre-circulation, approximately 100 hours of constant heat injection followed. More details about the borehole and both tests are provided in 47. 49 .

In the present work, as it is focused in modelling the thermal response of the BHE in the short-term, only the short-term experimental measurements will be considered. Therefore, for the model validation, just the first hours of heat injection of each test are considered. During this interval, the experimental measurements of the outlet water temperature exiting the BHE as well as the water temperature profile inside the $\mathrm{U}$ pipe, are used for the validation. 
3.2. TRNSYS simulation

The B2G has been validated against experimental data from the borehole described in section 3.1. TRNSYS simulation software has been chosen for performing the required simulations. The model has been implemented creating a new type in which all parameters of the thermal network are introduced as inputs, as well as the inlet water temperature and mass flow rate in the BHE. The BHE outlet water temperature is an output of the simulation. Additionally, the water temperatures at any given depth for each simulation time step are saved in a text file that can be subsequently analyzed. MATLAB software has been used for the analysis of the vertical temperature profiles inside the BHE.

The results are double validated using experimental data from two different step-tests with different operating conditions. Initially, only 10 hours of heat injection were simulated. The aim of this first analysis is to demonstrate the ability of the $\mathrm{B} 2 \mathrm{G}$ model to reproduce not only the outlet temperature evolution but also the internal temperature distribution at the U-pipe.

Once the accuracy of the model has been validated in a short-term basis (0 to 10 hours), the simulation time is extended, providing a medium-term validation (from 10 to 48 hours). Finally, the position of the grout nodes is analyzed and a sensitivity study on the values of this parameter is presented.

In the calculation of the model parameters, as explained in section 2.2 . the following assumptions have been made:

- An effective thermal conductivity of the water inside the borehole has been considered in order to take into account the convection phenomena happening during the heat injection. The effective water thermal 
conductivity has been estimated taking into account the experimental borehole thermal resistance, using equation 16 . For the $D_{e q}$, the Pasquier approach has been used (equation 15).

- Taking into account the dimensions of the borehole and the proximity of the pipes to the borehole wall, the grout nodes have been initially located on the borehole wall (although a sensitivity analysis of the model performance with different grout node positions is presented at the end of this section).

- The penetration depth has been adjusted in order to obtain a good prediction of the outlet water temperature at the end of the simulation time period considered. For the 10 hours simulation, the value of the penetration depth is around four times higher than the borehole diameter. However, as it can be observed in figure 9, the resulting adjusted values may vary between simulations for different heat injection durations. Since the penetration diameter does not appear as parameter on the model implementation, the adjustment has been performed by varying the value of the ground node thermal capacitance, and both the penetration depth and the corresponding thermal resistance are calculated from this value, following Eqs. 20 and 21. Finally, a least square error analysis has been conducted in order to determine the best fitting value for this parameter.

- The values of the rest of the model parameters have been calculated from the theoretical approach described in section 2.2 .

The B2G parameters considered in the present work are shown in Table1. 
343 (note that the thermal capacitances and the thermal resistances correspond

\section{Results and discussion}

\subsection{Step-test (short-term)}

Figure 5 shows the simulation results for the step-tests during the first 10 hours of heat injection in comparison with the experimental measurements. The simulated outlet temperature (continuous line) can thus be compared with the field measurements (dashed line). The parameters considered in the adjustment are shown in Table 1. Looking at Figure 5, it can be highlighted that for both step-tests, B2G is able to reproduce the outlet temperature profile with a good agreement. In particular, Figure 6 shows the deviation between the simulated and the experimental outlet water temperature profiles for both Test $1(\sqrt{6})$ and Test $2(6 \mathrm{~b})$, showing an absolute error within $0.15^{\circ} \mathrm{C}$.

In order to further investigate the performance of the model, the comparison is extended to the internal borehole temperature profiles. Figure 7 a shows the evolution of the water temperature for different depth nodes during the first 10 hours of injection in the first step-test. Moreover, Figure $7 \mathrm{~b}$ shows the vertical temperature profiles along the tube at different simulation periods. As it can be observed, B2G reproduces fairly well the internal temperature distribution with only a little discrepancy due to the vertical heat transfer phenomena associated to the step propagation which are neglected in the model (observed on Figure $7 \mathrm{~b}$, line 400 minutes).

The same comparison has been performed for the second step-test and similar results were obtained. In fact, both Figure $8 \mathrm{a}$ and Figure $8 \mathrm{~b}$ show a 


\begin{tabular}{|c|c|c|c|}
\hline \multicolumn{4}{|c|}{ Thermophysical properties } \\
\hline Ground thermal conductivity & $k_{g}$ & 3.1 & $W m^{-1} K^{-1}$ \\
\hline Grout thermal conductivity & $k_{b}$ & 1.675 & $W m^{-1} K^{-1}$ \\
\hline Ground volumetric thermal capacitance & $c_{g}$ & 2160 & $k J m^{-3} K^{-1}$ \\
\hline Grout volumetric thermal capacitance & $c_{b}$ & 4186 & $k J m^{-3} K^{-1}$ \\
\hline Ground thermal diffusivity & $\alpha_{g}$ & 0.005167 & $m^{2} h^{-1}$ \\
\hline Experimental mean borehole thermal resistance & $R_{b l}$ & 0.062 & $m K W^{-1}$ \\
\hline \multicolumn{4}{|c|}{ Geometrical characteristics } \\
\hline Borehole diameter & $D_{b}$ & 140 & $\mathrm{~mm}$ \\
\hline External U-pipe diameter & $D_{p, e}$ & 40 & $\mathrm{~mm}$ \\
\hline Internal U-pipe diameter & $D_{p, i}$ & 35.2 & $\mathrm{~mm}$ \\
\hline Shank spacing (center-to-center) & $W$ & 75 & $\mathrm{~mm}$ \\
\hline Depth & $L$ & 260 & $\mathrm{~m}$ \\
\hline \multicolumn{4}{|c|}{ Model parameters } \\
\hline Number of nodes & $\mathrm{n}$ & 254 & - \\
\hline Grout node thermal capacitance & $C_{b 1}-C_{b 2}$ & 53.73 & $J K^{-1}$ \\
\hline Ground node thermal capacitance & $C_{g}$ & 1100 & $J K^{-1}$ \\
\hline Borehole conductive thermal resistance & $R_{b 1}-R_{b 2}$ & 0.06131 & $K W^{-1}$ \\
\hline Pipe to pipe thermal resistance & $R_{p p}$ & 0.2910 & $K W^{-1}$ \\
\hline Grout to grout thermal resistance & $R_{b b}$ & 0.2389 & $K W^{-1}$ \\
\hline Grout to ground thermal resistance & $R_{g}$ & 0.05075 & $K W^{-1}$ \\
\hline Equivalent pipes diameter & $D_{e q}$ & 74.65 & $\mathrm{~mm}$ \\
\hline Grout node position & $D_{x}$ & 140 & $\mathrm{~mm}$ \\
\hline Ground radial penetration diameter & $D_{g p}$ & 593.6 & $\mathrm{~mm}$ \\
\hline Ground nodes position & $D_{g}$ & 366.8 & $\mathrm{~mm}$ \\
\hline
\end{tabular}

Table 1: Main parameter adopted (adju\&4tng for injection time equal to 10 hours). 
a)

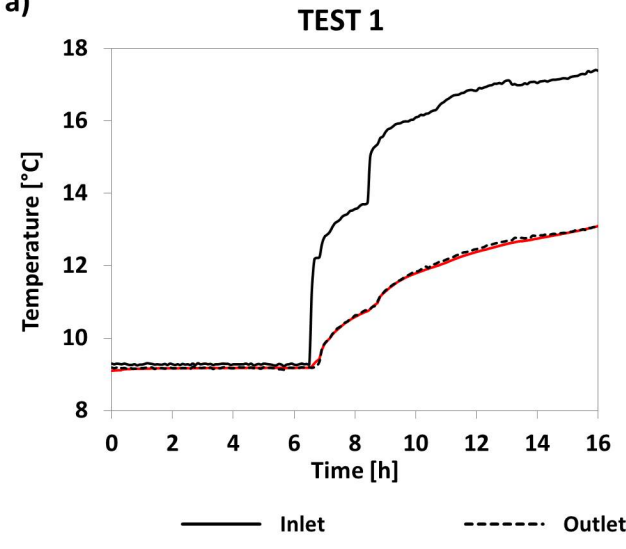

b)

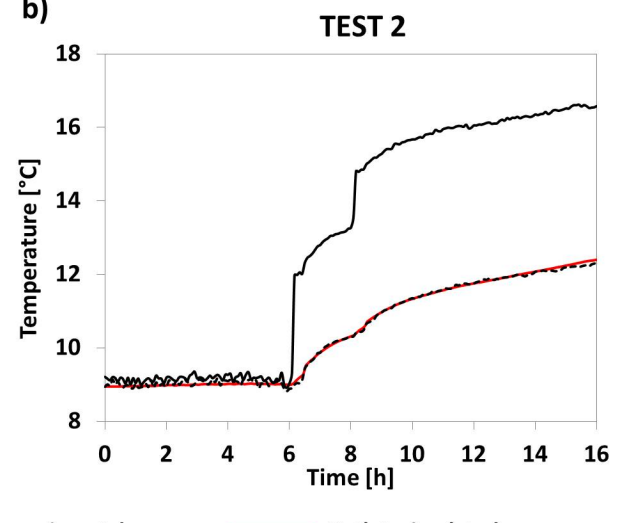

Figure 5: Comparison between the experimental and numerical outlet temperature for two different step-test.

very good agreement between the experimental and the numerical results.

The deviation shown in Figure 8b strictly depends on the initial conditions in which a little perturbation occurs.

In general, the prediction of the temperature profiles is accurate and the validation is considered successful.

\subsection{Step-test (extended time)}

In order to provide a medium-term validation, the simulation time has been extended taking into account greater injection times. An increase in the injection time has an influence on the volume of the ground affected by the borehole, which becomes greater as the injection time increases, making it necessary to consider a greater ground thermal capacitance in the model. This effect is solely connected with the penetration radius of the heat injection in the ground, which mainly depends on the thermo-physical characteristics of the ground. 


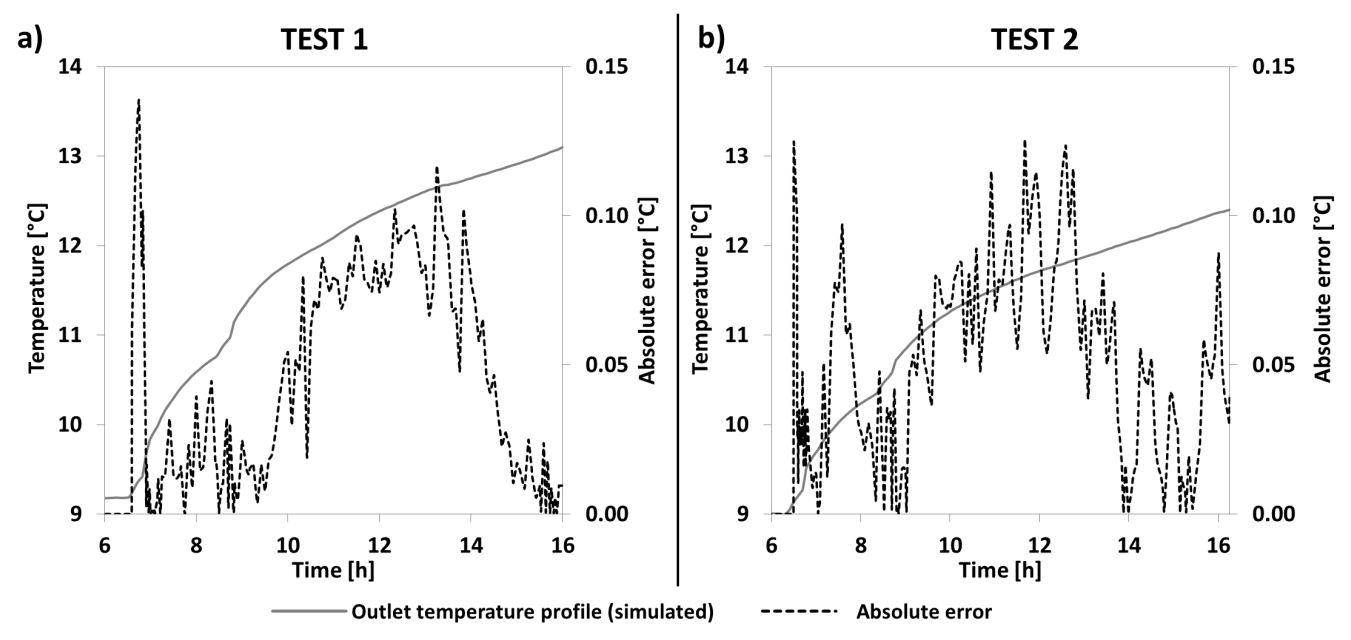

Figure 6: Absolute error between simulated and experimental outlet water temperature profiles: a) Test 1 ; b) Test 2 .

Figure 9 shows the comparisons between the experimental and numerical profiles of the outlet water temperature at the BHE considering different heat injection times. Starting from the adjustment provided in section 4.1 (for about 10h of injection), three different heat injection periods have been considered: 18 hours (Figure 9b), 30 hours (Figure 9k) and 42 hours (Figure 9d). The resulting ground parameters corresponding to each adjustment are reported in Table 2. Figures 9a to 9d show the results obtained with the initial 10 hours adjustment and the results obtained by readjusting the thermal capacitance of the ground node, respectively. As it can be observed, it is possible to obtain an acceptable medium-term adjustment by increas2 ing the ground thermal capacitance taking into account the greater ground volume affected. With the 10 hours adjustment, the model presents a lower 4 thermal inertia, leading to an increase of the outlet water temperature with 5 higher heat injection times. Higher values of the thermal capacitance of 
a)

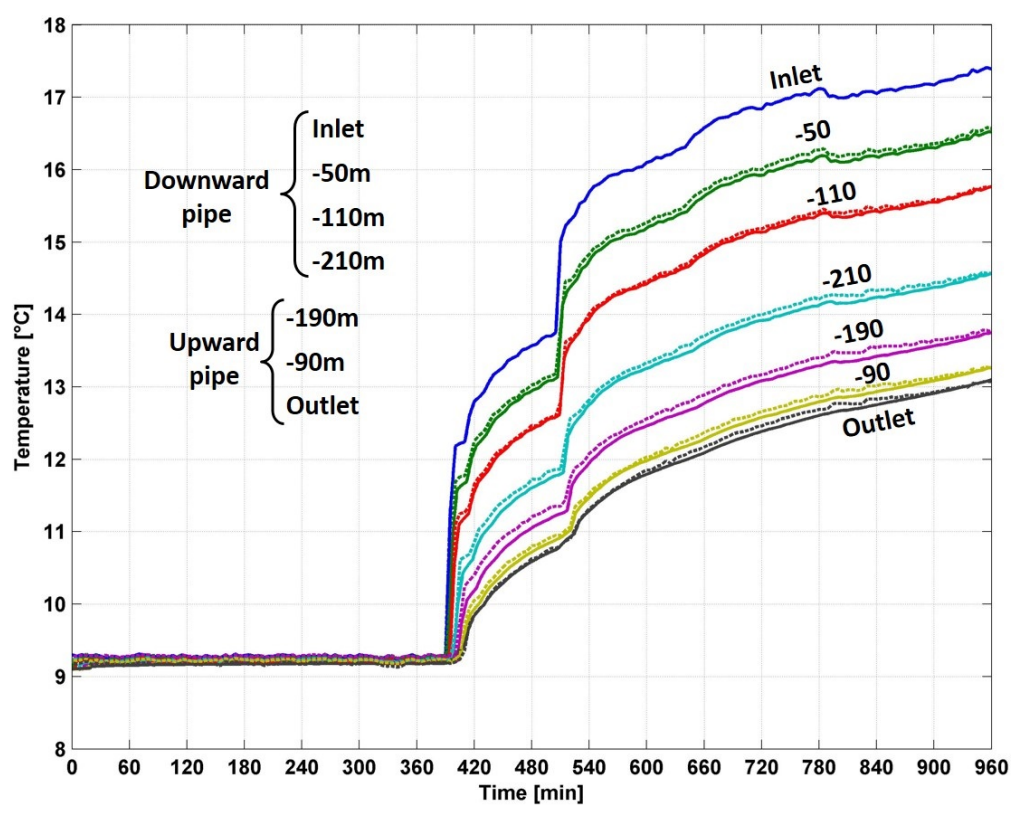

b)

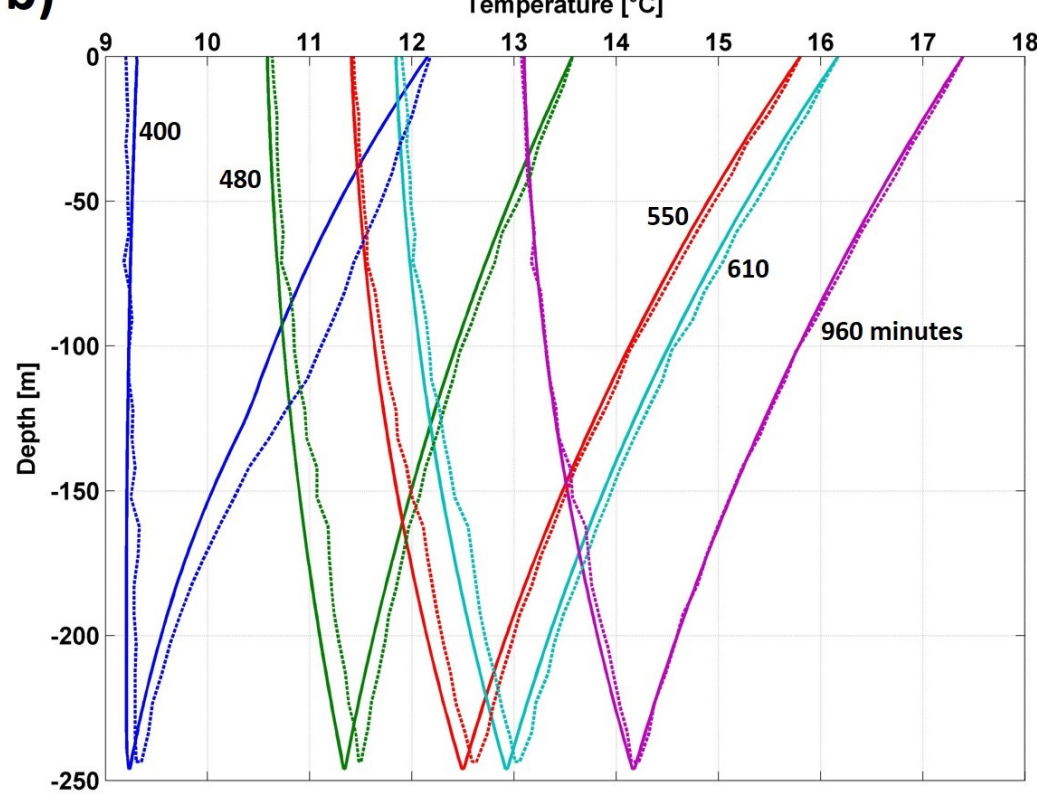

Figure 7: Comparison between the experimental (dashed lines) and numerical (continuous line) internal temperature profiles for the first step-test: a) temperature evolution at different depths. b) vertical profile at different simulation periods. 

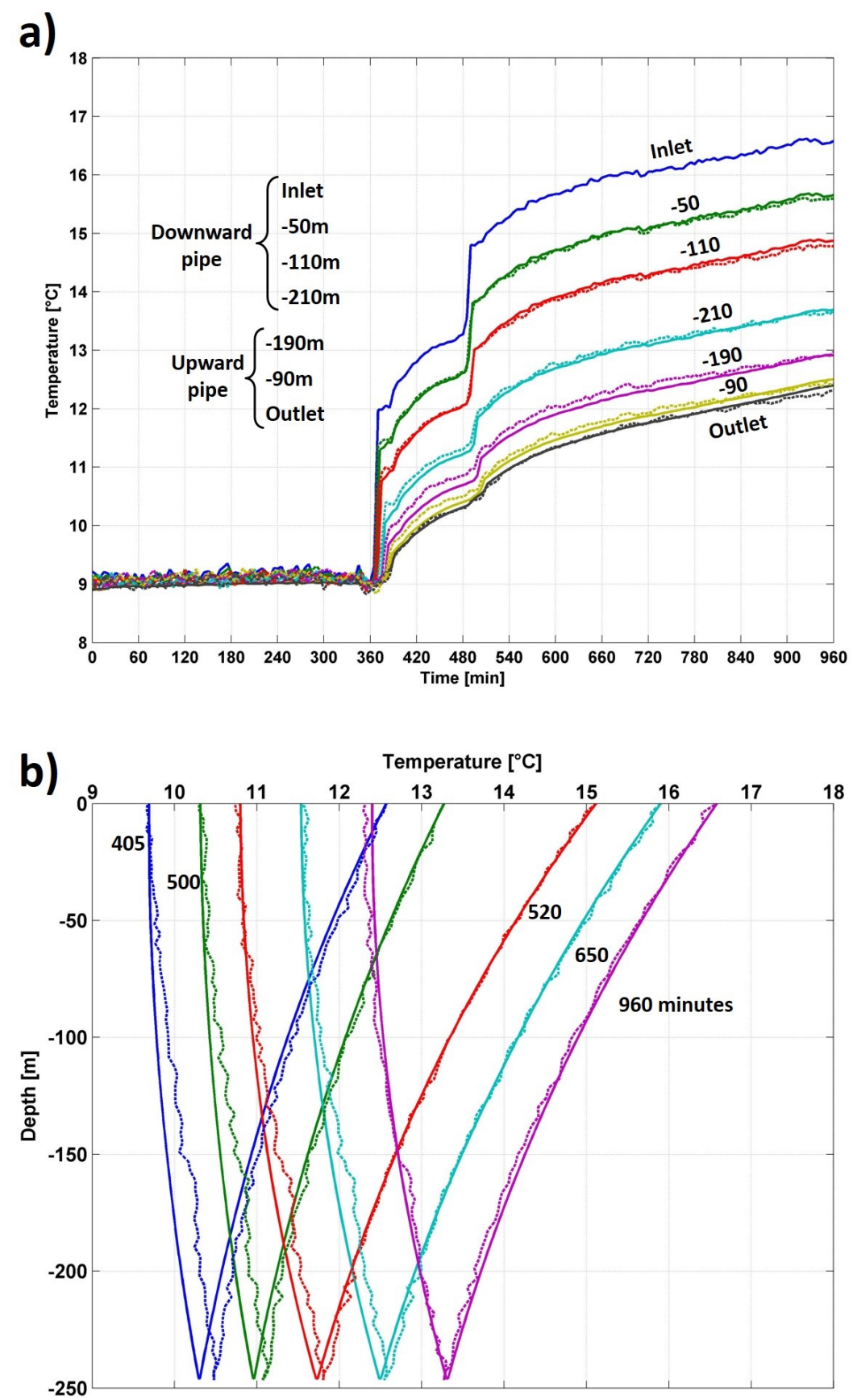

Figure 8: Comparison between the experimental (dashed lines) and numerical (continuous line) internal temperature profiles for the second step-test: a) temperature evolution at different depths. b) vertical profile at dif8rent simulation periods. 
the ground node produce a better adjustment in the medium-term by increasing the thermal inertia of the model. In particular, for lower injection times (Figure 9a,b), B2G is able to reproduce both the short-term and the medium-term behavior, once the thermal capacitance has been adjusted accordingly. Instead, for higher injection times (Figure 9k,d) it is possible to achieve a good accuracy in the medium-term response, but the higher thermal inertia needed in this case results in a higher difference in the short-term adjustment.

The results shown in Figure 9 prove that B2G results can be adjusted in order to obtain an accurate prediction of the medium-term temperature evolution for different heat injection times by only modifying the position of the ground node (and thus, the associated thermal capacitance and thermal resistances). In any case, since the $\mathrm{B} 2 \mathrm{G}$ aim is to reproduce the borehole behavior in a short-term basis, the same LSE analysis performed in order to determine the ground node parameters proves that the best results for this period are obtained with the 10 hours adjustment. For the 18 hours adjustment, the error is still acceptable, although not being minimum. However, the results obtained when adjusting the medium-term responses for 30 and 42 hours of heat injection are not acceptable for this specific purpose. Therefore, it can be concluded that B2G has proved to be useful for predicting the short-term behavior and the instantaneous response of the ${ }_{417}$ borehole for heat injection periods up to 18 hours, which is long enough for 8 the aim of this work. 


\begin{tabular}{|c|c|c|c|}
\hline \multicolumn{4}{|c|}{ Model parameters for 18 hours } \\
\hline Ground node thermal capacitance & $C_{g}$ & 2100 & $J K^{-1}$ \\
\hline Borehole to ground thermal resistance & $R_{g}$ & 0.06432 & $K W^{-1}$ \\
\hline Ground radial penetration diameter & $D_{g p}$ & 809.2 & $\mathrm{~mm}$ \\
\hline Ground nodes position & $D_{g}$ & 474.6 & $\mathrm{~mm}$ \\
\hline \multicolumn{4}{|c|}{ Model parameters for 30 hours } \\
\hline Ground node thermal capacitance & $C_{g}$ & 3550 & $J K^{-1}$ \\
\hline Borehole to ground thermal resistance & $R_{g}$ & 0.07604 & $K W^{-1}$ \\
\hline Ground radial penetration diameter & $D_{g p}$ & 1046 & $\mathrm{~mm}$ \\
\hline Ground nodes position & $D_{g}$ & 592.8 & $\mathrm{~mm}$ \\
\hline \multicolumn{4}{|c|}{ Model parameters for 42 hours } \\
\hline Ground node thermal capacitance & $C_{g}$ & 5000 & $J K^{-1}$ \\
\hline Borehole to ground thermal resistance & $R_{g}$ & 0.05251 & $K W^{-1}$ \\
\hline Ground radial penetration diameter & $D_{g p}$ & 1238 & $\mathrm{~mm}$ \\
\hline Ground nodes position & $D_{g}$ & 688.9 & $\mathrm{~mm}$ \\
\hline
\end{tabular}

Table 2: Node ground parameter adopted for different injection times (note that thermal capacitances and resistances are node values and, as a consequence, they depend on the number of nodes adopted). 

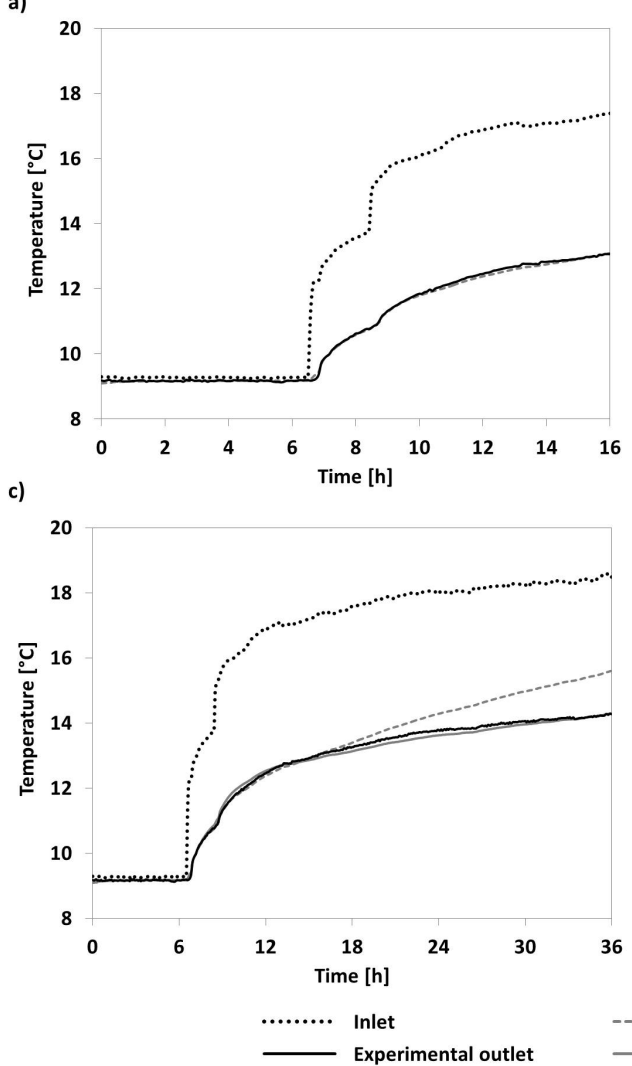

b)

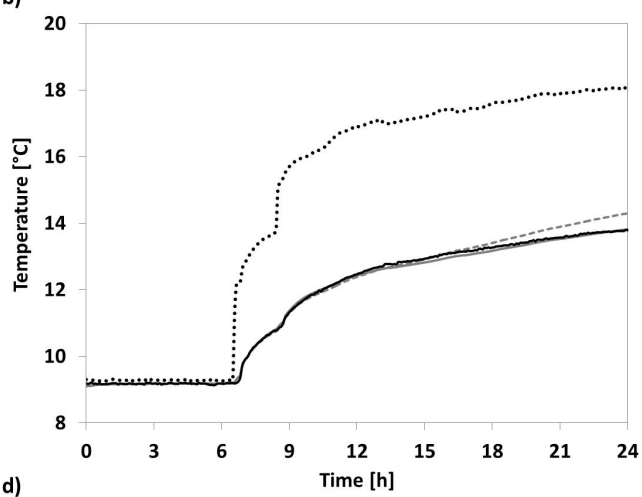

d)

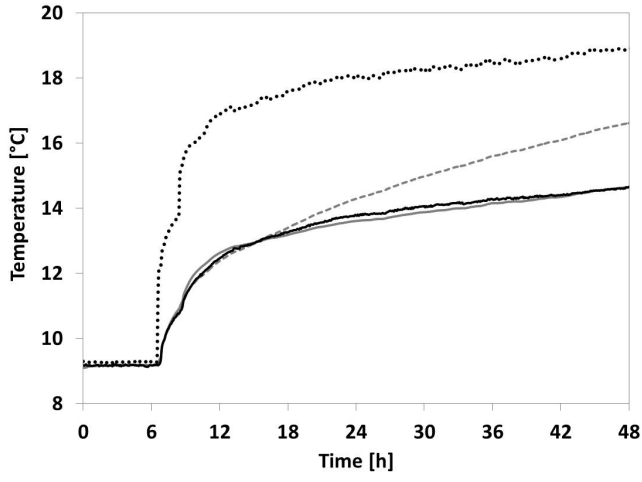

Figure 9: Comparison of the results obtained for different heat injection periods (in hour): a) 10; b) 18 ; c) 30 ; d) 42 .

\section{3. $D_{x}$ analysis}

The analysis of the position of the grout nodes has been performed using the 10 hours simulation adjustment. Considering that the grout nodes have to be located somewhere between $D_{e q}$ and $D_{b}$, as stated in section 2.2 , the value of $D_{x}$ can be calculated by Eq. 29 .

$$
D_{x}=a\left(D_{b}\right)+(1-a) D_{e q} \quad \text { with } 0<a<1
$$




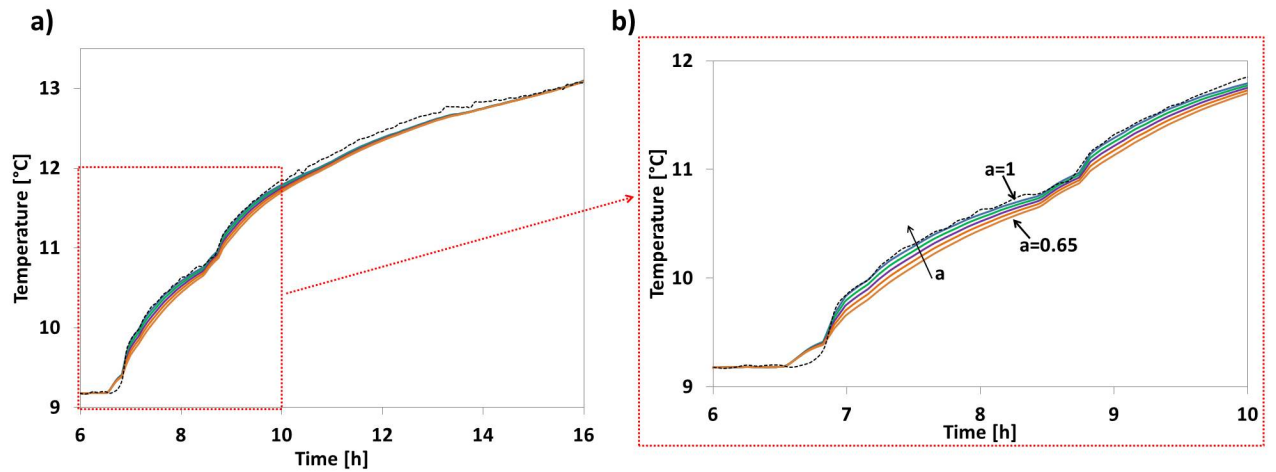

Figure 10: Simulation results with different values of $D_{x}$.

431 11. It can be observed in this figure that the position of the borehole tal outlet water temperature. Different values of $D_{x}$ have been considered, corresponding to different values of the parameter $a$ of $1\left(D_{x}=D_{b}\right), 0.92$, $0.83,0.75$, and 0.65 . The simulation time corresponds to a total heat injection time of about 10 hours, as shown in Figure 10a. Figure 10b shows an augmented view of the first hours of the step, in order to highlight the differences between the different simulation results.

Results show that the best fitting is obtained locating the grout nodes at the borehole wall, validating the initial assumption made in this work. The absolute errors between simulated and experimental outlet temperature profiles of TEST 1 for different grout node positions are plotted in Figure ${ }_{32}$ nodes mainly affects the instantaneous response of the model: for this type of BHE, the absolute error tends to increase if the value of $a$ decreases. However, as it can be observed in Figure 11a, values of $a$ between 0.8 and 1 also produce valid results (i.e. absolute error within $0.15^{\circ} \mathrm{C}$ ). On the other

Figure 10 plots the simulation results for the simulated and experimen- 


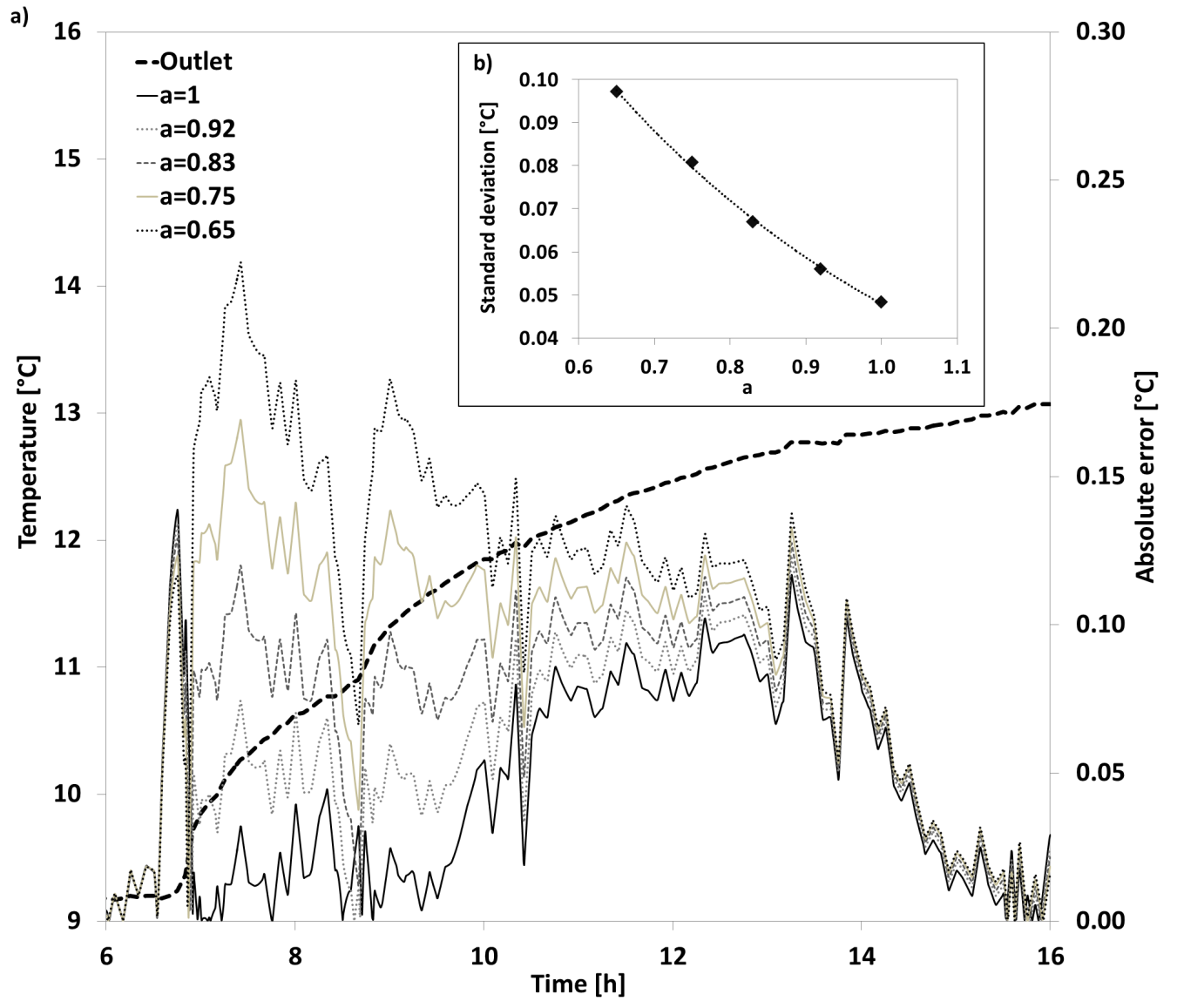

Figure 11: a) Absolute error between simulated and experimental outlet water temperature profiles of TEST 1 for different grout node positions. b) Standard deviation against parameter $a$. 
hand, results corresponding to $a$ values lower than 0.8 fall too far from the experimental ones to be considered valid for the short-term simulation. The same result is confirmed also in Figure $11 \mathrm{~b}$, where the standard deviation between simulated and experimental data is plotted against the parameter $a$ : a general decrease of the standard deviation is observed for grout node locations closer to the borehole diameter.

Therefore, it can be concluded that, for the proposed model and for the borehole studied in this work, the position of the grout nodes needs to be considered near the borehole wall, becoming the assumption of the borehole wall itself the best option.

\section{Conclusions}

A novel BHE model, called Borehole-to-Ground (B2G) model, was presented and validated against experimental data from a real borehole. When modelling complete GSHP systems, it i necessary to use models with low computational costs. In order to obtain low computational costs for the GSHE model, the short-term and long-term responses were decoupled. B2G was intended to reproduce only the short-term response of the borehole outlet fluid temperature for heat injection pulses up to 10-15 hours long, which corresponds to the total injection time each day on a typical installation.

The proposed $\mathrm{B} 2 \mathrm{G}$ model was double validated against experimental data from two different step tests of the same borehole, for a heat injection time of about 10 hours. Results show that B2G is able to reproduce the outlet temperature profile with an agreement within a $0.1 \mathrm{~K}$ difference. Together with the outlet fluid temperature, the internal temperature profiles along the U-tube were also compared, showing a good agreement with the 
471 for this BHE configuration.

472 Finally, the calculation of the parameters of B2G has proven to be quite 473 straightforward, starting from the overall borehole thermal resistance and 474 its geometrical an thermal properties. This simplifies the use of B2G for 475

${ }_{477}$ The present work has been supported by the FP7 European project 478 "Advanced ground source heat pump systems for heating and cooling in 479 Mediterranean climate" (GROUND-MED), and by the "Resource-Efficient 480

experimental measurements.

Two main parameters of adjustment were identified in the model: the penetration depth and the position of the grout nodes. In order to determine these parameters, two sensitivity analyses were carried out: (i) study of the influence of the heat injection period on the penetration depth, and (ii) impact of the grout nodes position on the B2G performance. The results obtained in (i) showed that it is possible to adjust the model to different heat injection periods by varying the ground node position (i.e. the radial penetration depth). On the other hand, results from (ii) showed that locatBHE short-term simulation, making it a user-friendly simulation tool.

\section{Acknowledgements}

$$
\text { Refrigeration And Heat Pump Systems" (EFFSYS+) program. }
$$




\begin{tabular}{|c|c|c|c|}
\hline \multicolumn{4}{|c|}{ Nomenclature } \\
\hline$\alpha$ & Thermal diffusivity $\left[\mathrm{m}^{2} / \mathrm{s}\right]$ & & oscripts \\
\hline $\mathrm{BHE}$ & Borehole heat exchanger & & Downward pipe zone \\
\hline $\mathrm{c}$ & Volumetric thermal capacity $\left[\mathrm{J} / \mathrm{m}^{3} \mathrm{~K}\right]$ & 2 & Upward pipe zone \\
\hline $\mathrm{C}$ & Thermal capacitance $[\mathrm{J} / \mathrm{K}]$ & $\mathrm{b}$ & borehole grout \\
\hline $\mathrm{D}$ & diameter $[\mathrm{m}]$ & $\mathrm{bb}$ & borehole grout to borehole grout \\
\hline GSHE & Ground source heat exchanger & $\mathrm{c}$ & conductive \\
\hline GSHP & Ground source heat pump & e & external \\
\hline $\mathrm{k}$ & conductivity $[\mathrm{W} / \mathrm{mK}]$ & $\mathrm{EC}$ & External circuit (ground loop) \\
\hline $\mathrm{h}$ & convective heat transfer coefficient $\left[\mathrm{W} / \mathrm{m}^{2} \mathrm{~K}\right]$ & eq & equivalent \\
\hline $\mathrm{L}$ & depth $[\mathrm{m}]$ & $\mathrm{g}$ & ground \\
\hline$\dot{m}$ & Mass flow rate $[\mathrm{kg} / \mathrm{h}]$ & gp & ground penetration \\
\hline $\mathrm{n}$ & number of nodes [-] & $\mathrm{j}$ & j-node \\
\hline $\mathrm{Nu}$ & Nusselt number [-] & $\mathrm{h}$ & convection \\
\hline $\mathrm{r}$ & radius $[\mathrm{m}]$ & $\mathrm{i}$ & internal \\
\hline $\mathrm{R}$ & Thermal resistance $[\mathrm{K} / \mathrm{W}]$ & $\mathrm{IC}$ & Internal circuit (building) \\
\hline $\mathrm{R}_{B H E}$ & Borehole thermal resistance $[\mathrm{mK} / \mathrm{W}]$ & in & Inlet \\
\hline $\mathrm{R}_{12}$ & Fluid to fluid thermal resistance $[\mathrm{mK} / \mathrm{W}]$ & $\mathrm{p}$ & pipe \\
\hline $\mathrm{S}$ & surface area $\left[\mathrm{m}^{2}\right]$ & $\mathrm{pp}$ & pipe node to pipe node \\
\hline $\mathrm{t}$ & Time $[s]$ & out & Outlet \\
\hline $\mathrm{T}$ & Temperature $[\mathrm{C}]$ & $\mathrm{x}$ & borehole node position \\
\hline $\mathrm{v}$ & velocity $[\mathrm{m} / \mathrm{s}]$ & & \\
\hline W & shank spacing $[\mathrm{m}]$ & & \\
\hline $\mathrm{Z}$ & Borehole depth coordinate $[\mathrm{m}]$ & & \\
\hline
\end{tabular}


[1] Environmental Protection Agency: http://www.epa.gov/region1/eco/energy/re_geothermal.html, 14/03/2013.

[2] Florides, G., Kalogirou, S., 2007. Ground heat exchangers - A review of systems, models and applications. Renewable Energy 32, 2461-2478.

[3] Ozgener, O., Hepbasli, A., 2005. IPerformance analysis of a solar-assisted groundsource heat pump system for greenhouse heating:an experimental study. Building and Environment 40, 1040-1050.

[4] Sharqawy, M.H., Mokheimer, E.M., Badr, H.M., 2009. Effective pipe-to-borehole thermal resistance for vertical ground heat exchangers. Geothermics 38, 271-277.

[5] Esen, H., Inalli, M., 2009. Modelling of a vertical ground coupled heat pump system by using artificial neural networks. Expert Systems with Applications 36, 1022910238.

[6] Oppelt, T., Riehl, I., Gross, U., 2010. Modelling of the borehole filling of double U-pipe heat exchangers. Geothermics 39, 270-276.

[7] Corberán, J.M., Finn, D.P., Montagud, C., Murphy, F.T., Edwards, K.C., 2011. A quasi-steady state mathematical model of an integrated ground source heat pump for building space control. Energy and Buildings 43, 82-92.

[8] Bak, K., Ozyurt, O., Comakli, K., Comakli, O., 2011. Energy analysis of a solarground source heat pump system with vertical closed-loop for heating applications. Energy 36, 3224-3232.

[9] Montagud, C., Corberán, J.M ., Montero, Á., Urchueguía, J.F., 2011. Analysis of the energy performance of a Ground Source Heat Pump system after five years of operation. Energy and Buildings 43, 3618-3626.

[10] Sagia, Z., Rakopoulos, C., Kakaras, E. 2012. Cooling dominated Hybrid Ground Source Heat Pump System application. Applied Energy 94, 41-47.

[11] Man, Y., Yang, H., Wang, J. 2012. In situ operation performance test of ground coupled heat pump system for cooling and heating provision in temperate zone. Applied Energy 97, 913-920.

[12] Eslami-nejad, P., Bernier, M. 2012. Freezing of geothermal borehole surroundings: A numerical and experimental assessment with applications. Applied Energy 98, $333-345$. 
[13] Capozza, A., De Carli, M., Zarrella, A. 2013. Investigations on the influence of aquifers on the ground temperature in ground-source heat pump operation. Applied Energy 107, 350-363.

[14] Go, G.H., Lee, S.R., Yoon, S., Kang, H., 2014. Design of spiral coil PHC energy pile considering effective borehole thermal resistance and groundwater advection effects. Applied Energy 125, 165-178.

[15] Yang, H., Cui, P., Fang, Z., 2010. Vertical-borehole ground-coupled heat pumps: A review of models and systems. Applied Energy 87, 16-27.

[16] Ingersoll, L.R., Plass, H.J., 1948. Theory of the ground pipe source for the heat pump. ASHVE Trans 54, 339-348.

[17] Ingersoll, L.R., Adler, F.T., Plass, H.J. 1950. Theory of earth heat exchangers for the heat pump. ASHVE Trans 56, 16788.

[18] Carslaw, H.S., Jaeger, J.C. 1946. Conduction of heat in solids. Oxford UK: Claremore Press.

[19] Eskilson, P., 1987. Thermal analysis of heat extraction boreholes. PhD Thesis, University of Lund, Sweden.

[20] Michopoulos, A., Kyriakis, N., (2009). Predicting the fluid temperature at the exit of the vertical ground het exchangers. Applied Energy 86, 2065-2070

[21] Yavuzturk, C., Spitler, J.D., 1999. A Short Time Step Response Factor Model for Vertical Ground Loop Heat Exchangers. ASHRAE Transactions 105(2), 475-485.

[22] Spitler, J.D., 2000. GLHEPRO - A Design Tool For Commercial Building Ground Loop Heat Exchangers. Proceedings of the Fourth International Heat Pumps in Cold Climates Conference, Aylmer, Qubec. August 17-18.

[23] Hellstrom G, Sanner B. Earth energy designer: software for dimensioning of deep boreholes for heat extraction. Sweden: Department of Mathematical Physics, Lund University; 1994.

[24] Monzó, P., Mogensen, P., Acuña, J., 2014. A novel numerical model for the thermal response of borehole heat exchanger fields. 11th IEA Heat Pump Conference, Montréal, Canada.

[25] Eskilson P, Claesson J. Simulation model for thermally interacting heat extraction boreholes. Numerical Heat Transfer. 1988; 13:149-65. 
[26] Yang, H., Cui, c., Fang, Z., 2010. A two-region simulation model of vertical Utube ground heat exchanger and its experimental verification. Applied Energy 86, 2005-2012.

[27] Javed, S., Claesson, J., 2011. New analytical and numerical solutions for the shortterm analysis of vertical ground heat exchangers. ASHRAE Transactions, vol.17(1), $3-12$.

[28] Monteyne, G., Javed, S., Vandersteen G., 2014. Heat transfer in a borehole heat exchanger: Frequency domain modeling. International Journal of Heat and Mass Transfer, 69, 129-139.

[29] Li, M., Lai, A.C.K., 2012. New temperature response functions (G functions) for pile and borehole ground heat exchangers based on composite-medium line-source theory. Energy, 38, 255-263.

[30] Li, M., Lai, A.C.K., 2012. Analytical model for short-time responses of ground heat exchangers with U-shaped tubes: Model development and validation. Applied Energy, 104, 510-516.

[31] Bauer, D., Heidemann, W., Müller-Steinhagen, H., Diersch, H.-J. G., 2011. Thermal resistance and capacity models for borehole heat exchangers. Intenational Journal of Energy Research, 35:312320.

[32] Bauer, D., Heidemann, W., Diersch, H.-J.G., 2011. Transient 3D analysis of borehole heat exchanger modeling. Geothermics 40, 250-260.

[33] Pasquier, P., Marcotte, D., 2012. Short-term simulation of ground heat exchanger with an improved TRCM. Renewable Energy 46, 92-99.

[34] Lamarche, L., Kajl, S., Beauchamp, B., 2010. A review of methods to evaluate borehole thermal resistances in geothermal heat-pump systems. Geothermics 39, 187-200.

[35] Diersch, H.-J.G., Bauer, D., Heidemann, W., Rühaak, W., Schätzl, P., 2011. Finite element modeling of borehole heat exchanger systems Part 1. Fundamentals. Computers \& Geosciences 37, 1122-1135.

[36] Diersch, H.-J.G., Bauer, D., Heidemann, W., Rühaak, W., Schätzl, P., 2011. Finite element modeling of borehole heat exchanger systems Part 2. Numerical simulation. Computers \& Geosciences 37, 1136-1147. 
[37] Esen, H., Inalli, M., Esen, Y., 2009. Temperature distributions in boreholes of a vertical ground-coupled heat pump system. Renewable Energy 34, 2672-2679.

[38] Lee, C.K., Lam, H.N., 2008. Computer simulation of borehole ground heat exchangers for geothermal heat pump systems. Renewable Energy 33, 1286-1296.

[39] Koohi-Fayegh, S., Rosen, M.A., 2012. Examination of thermal interaction of multiple vertical ground heat exchangers. Applied Energy 97, 962-969.

[40] Florides, G.A., Christodoulides, P., Pouloupatis, P., 2012. An analysis of heat flow through a borehole heat exchanger validated model. Applied Energy 92, 523-533.

[41] Florides, G.A., Christodoulides, P., Pouloupatis, P., 2013. Single and double U-tube ground heat exchangers in multiple-layer substrates. Applied Energy 102, 364-373.

[42] Luo, J., Rohn, J., Bayer, M., Priess, A., Xiang, W., 2014. Analysis on performance of borehole heat exchanger in a layered subsurface. Applied Energy 123, 55-65.

[43] De Rosa, M., Ruiz-Calvo, F., Corberán, J.M., Montagud, C., Tagliafico, L.A., 2014. Borehole modelling: a comparison between a steady-state model and a novel dynamic model in a real ON/OFF GSHP operation. 32nd UIT Heat Transfer Conference, Pisa, June 2014.

[44] Zarrella, A., Scarpa, M., De Carli, M., 2011. Short time-step performances of coaxial and double U-tube borehole heat exchangers: Modeling and measurements. HVAC\&R Research, 17:6, 959-976.

[45] Gnielinsky, V., 1976. New equations for heat and mass transfer in turbulent pipe and channel flow. International Chemical Engineering 16, 359-368

[46] Lax. P., Wendroff. B., 1960. Systems of Conservation Laws. Communications on Pure and Applied Mathematics, vol. XIII, 217-237.

[47] Acuña, J., Mogensen, P., Palm, B. 2009. Distributed thermal response tests on a U-pipe borehole heat exchanger. Effstock - The 11th International Conference on Energy Storage, Stockholm, 2009.

[48] Acuña, J., Palm, B., Hill, P. 2008. Characterization of boreholes - results from a U-pipe borehole heat exchanger installation [Conference]. Zurich: IEA Heat Pump Conference, 4.19.2008.

[49] Acuña, J., 2013. Distributed thermal response tests - New insights on U-pipe and Coaxial heat exchangers in groundwater-filled boreholes. PhD thesis, KTH. 